\title{
Retour à Hienghène : une vallée calédonienne de la colonisation à l'espace post-colonial
}

\section{Alain Saussol}

\section{(2) OpenEdition Journals}

Édition électronique

URL : http://journals.openedition.org/jso/6303

DOI : $10.4000 /$ jso.6303

ISSN : $1760-7256$

Éditeur

Société des océanistes

Édition imprimée

Date de publication : 30 juin 2011

Pagination : 77-92

ISBN : 978-2-85430-030-7

ISSN : 0300-953x

Référence électronique

Alain Saussol, « Retour à Hienghène : une vallée calédonienne de la colonisation à l'espace postcolonial », Journal de la Société des Océanistes [En ligne], 132 | 1er semestre 2011, mis en ligne le 30 juin 2013, consulté le 30 avril 2019. URL : http://journals.openedition.org/jso/6303 ; DOI : 10.4000/ jso.6303 


\title{
Retour à Hienghène : une vallée calédonienne de la colonisation à l'espace post-colonial ${ }^{1}$
}

par

\author{
Alain SAUSSOL*
}

\section{RÉSUMÉ}

Dans le nord-est de la Nouvelle-Calédonie, le pays de Hienghène s'est trouvé confronté à une décolonisation soudaine et à la nécessité de reconstruire un système foncier et une organisation économique. Ancien pays vivrier mélanésien, il était devenu, à la fin du XIX siècle, un centre de colonisation européenne voué à la caféiculture. Cette emprise coloniale a connu son maximum d'extension vers le milieu de la décennie 1960. Prolongement des événements de 1968, à partir de 1970, la contestation kanak foncière et politique a fragilisé cette colonisation vieillissante. Avec l'explosion violente de 1984, les Kanak retrouvaient soudain le contrôle de leurs terres ancestrales. Quallaient-ils en faire et comment allaient-ils les répartir entre eux? Cet article analyse la voie suivie pour cette reconstruction avec l'invention d'un outil spécifique, pont entre coutume et modernité, le groupement de droit particulier local. Il montre aussi les changements économiques et sociaux qui ont accompagné la transformation de la vallée et de l'ensemble du pays de Hienghène, depuis un demi siècle.

Mots-ClÉs : Nouvelle-Calédonie, Kanak, décolonisation, coutume, reconstruction territoriale

Sur la côte nord-est de Nouvelle-Calédonie, à 400 kilomètres de la capitale Nouméa, la vallée de Hienghène a longtemps constitué un bout du monde. Étroite, encaissée dans son cadre mon-

\begin{abstract}
As a geographer, $A$. Saussol conducted several studies in the Valley of Hienghene between 1965 and 1970. At the time, it was a place of ageing, coffee-cropping European settlements, where Kanak were gathered in so called "native reserves," which had become insufficient. As a result, starting in 1970, protests arose on land issues, and then escalated on political matters. Tensions mounted. After a period of disorder, the conflict reached its paroxysm in 1984, marked by the slaughtering of Kanak militants, which led to the hasty (and final) departure of the settlers. The Kanak then regained full control over their ancestor's land. Will they, driven by respect for custom, re-establish the former pre-colonial society or will they organize a new post-colonial order founded on different bases? This is the issue considered by the author, who returned to Hienghene in 2004, as he follows the evolution in the valley over the previous half century.
\end{abstract}

Keywords: New Caledonia, Kanak, decolonization, custom, territorial reconstruction

1. J'exprime ma gratitude à tous ceux envers qui je suis redevable et particulièrement : J. Bouarate, J. Ty, E. Béalo, S. et H. Mayat, J.-F. Houaoulo, D. Kowi, J. et M. Couhia, S. Valiadimoin, M. Wahou, V. Tjibaou, B. Maépas, Ben Lahoussine, L. Mapou, A. Ponidja, P. Godin, I. Leblic, M. Campenet et M. Martotaroeno qui, jeune stagiaire frais émoulu de l'École de Port Laguerre, avait participé à mes enquêtes de 1967 à Hienghène.

* Alain Saussol, professeur émérite de géographie, aujourd'hui honoraire, de l'Université de Montpellier, asaussol@ wanadoo.fr 


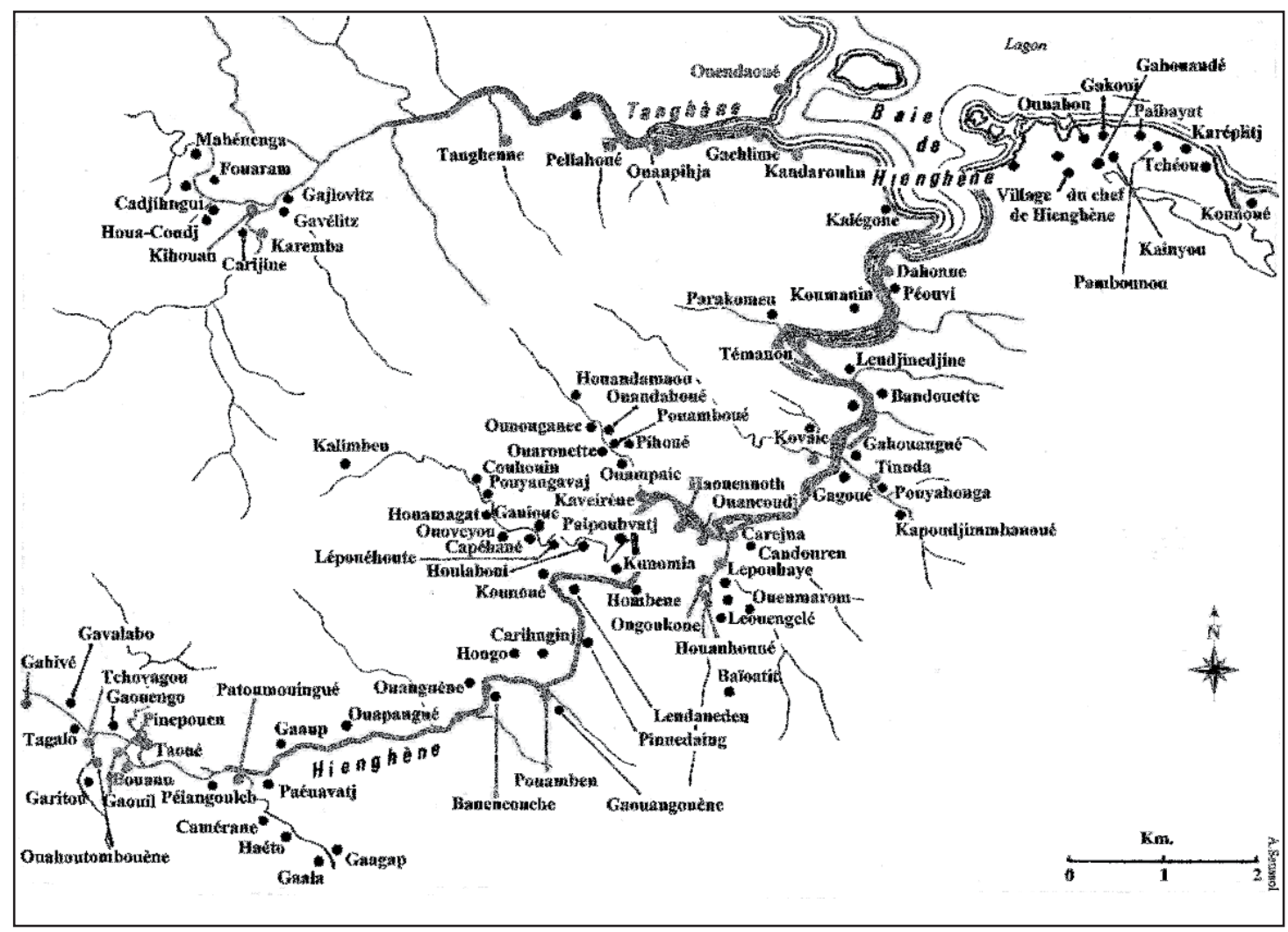

Figure 1. - Répartition des hameaux kanak précoloniaux dans les vallées de la Hienghène et de la Tanghène, vers 1878 (d'après les levés précédant le « cantonnement» des géomètres Engler et Ratzel)

Les noms des hameaux (et leur orthographe) sont ceux portés par les géomètres sur ces levés. On voit que ce pays, relativement peuplé, chichement doté en terres cultivables, était déjà largement occupé et n'aurait jamais dû être ouvert à la colonisation.

À l'arrivée des Européens, deux chefferies contrôlaient cet espace, celle de Bouarate (Bwarhat) au bord de mer et dans la moyenne vallée et celle de Goa dans la haute vallée. Le peuplement se répartissait en hameaux lignagers dispersés à l'abri des crues le long de la rivière et des ruisseaux affluents, sur les hautes terrasses, le piedmont ou les replats de la montagne. Leurs habitants pratiquaient une agriculture vivrière de gros tubercules, ignames et taros et la pêche sur le littoral. La propriété du sol, de type familial, était gérée par l'aîné du lignage descendant des ancêtres défricheurs. C'est à lui que revenait le soin de répartir les droits d'usage sur la terre qui restait un héritage commun. Fédérant l'ensemble des clans présents sur son territoire, ceux issus du pays et d'autres venus d'ailleurs, la chefferie, représentée par son tertre et sa grande case, constituait le niveau politique de l'organisation sociale.

\section{Hienghène à l'apogée de sa situation coloniale (fin des années 1960)}

Malgré sa population relativement nombreuse, sur le prétexte du déclin démographique des Mélanésiens, à la fin du XIX ${ }^{e}$ siècle cette vallée avait été ouverte à la colonisation. À la suite d'opérations de " cantonnement ", les Kanak avaient été refoulés et regroupés dans des " réserves indigènes » où la terre était présumée collective par décision de l'Administration coloniale (un non sens pour des paysans kanak d'autant plus attachés à leur sol familial qu'ils le tenaient de leurs ancêtres qui l'avaient défriché).

Sur les terres ainsi débarrassées de leurs premiers occupants, l'Administration délimita des concessions de 25 à 50 hectares (dont un quart seulement s'avérait cultivable) pour y installer un paysannat de petits colons français venus planter du café. Pendant un siècle, la vallée allait ainsi juxtaposer des propriétés coloniales toujours plus envahissantes et des " réserves" indigènes de plus en plus insuffisantes. À cela s'ajoutaient, dans la montagne ou en étroite frange le long du littoral, des espaces non affectés constituant le domaine public de la Colonie (dont une partie pouvait être louée aux éleveurs comme pâturage extensif). Telle était encore la situation, entre 1965 et 1967, lors d'un premier séjour dans cette région.

Revenu à Hienghène en 2004, je retrouvais une vallée très différente de celle parcourue un demi siècle plus tôt. Entre temps, au terme d'une san- 


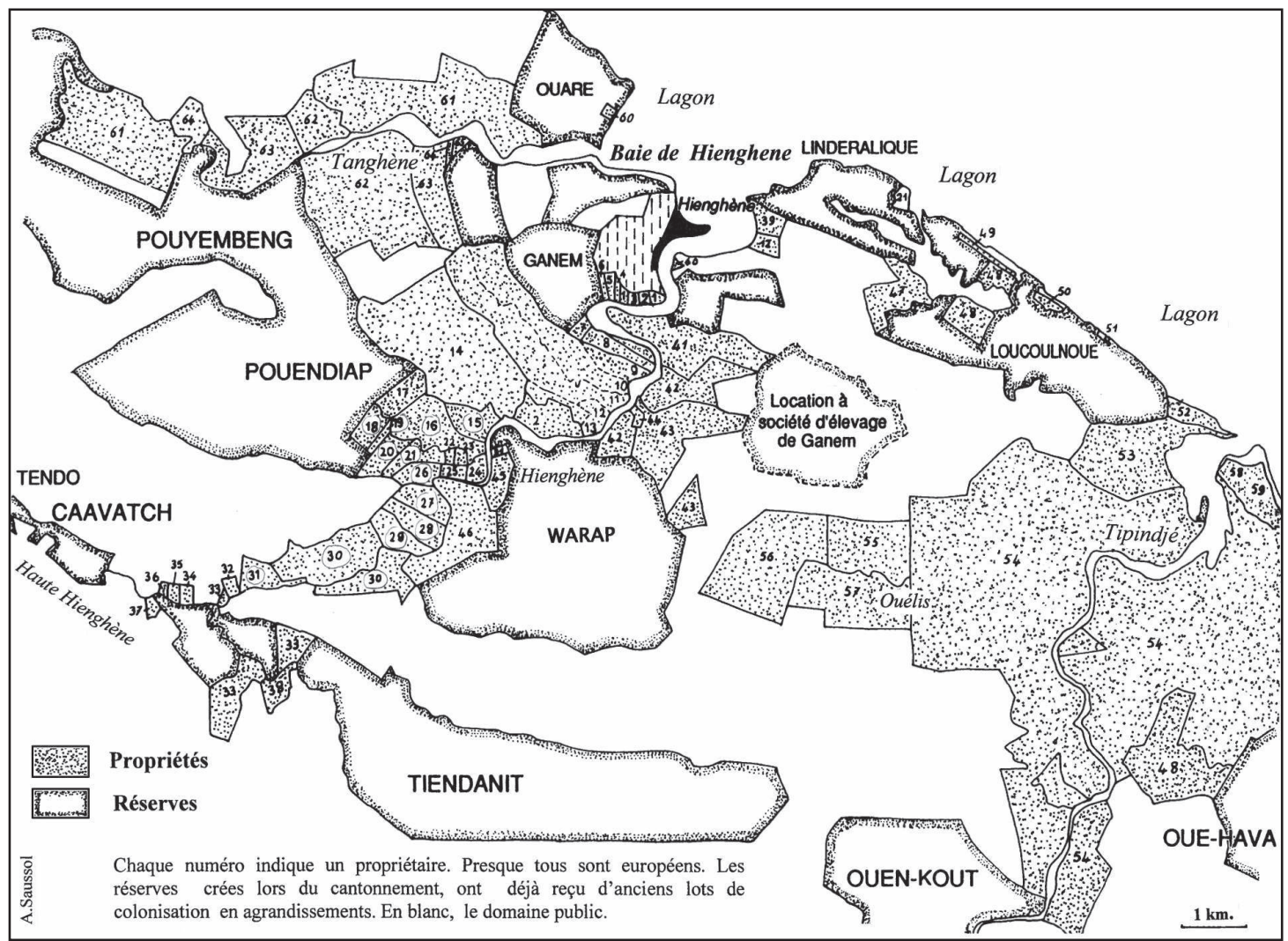

FIGURE 2. - Répartition des terres, en 1981, entre propriétés coloniales et "réserves indigènes ", dans les trois vallées de la Hienghène, de la Tanghène et de la Tipindjé

Cette carte montre comment se répartissaient les terres dans les dernières années de la présence coloniale à Hienghène. Les numéros inscrits sur les propriétés indiquent les propriétaires, un même numéro désignant un même propriétaire. À cette date, il y a trois ans que la réforme foncière a été engagée. Aucune rétrocession de terre n'a encore eu lieu. Pourtant $40 \%$ de la surface détenue par les Européens concerne des colons âgés de plus de 65 ans. On compte alors à Hienghène 200 Européens et 1500 Kanak groupés dans les "réserves ». Le climat est tendu. Trois ans plus tard éclatera le drame de Wanyatt.

glante tragédie, ayant rompu ses amarres avec le système colonial et tourné une page, le pays de Hienghène avait entrepris, non pas un renvoi à son lointain passé mais, par des voies différentes, un voyage sans retour. Expérience inédite dont cette note propose de rendre compte.

Entre 1965 et 1967, Hienghène était à l'apogée de sa situation coloniale. Deux cents Européens et presque autant de Javanais y côtoyaient 1330 Kanak. Les colons détenaient la majorité des terres alluviales. Leurs concessions occupaient l'essentiel de la vallée remontant jusqu'à une quinzaine de kilomètres du littoral. Dans l'ensemble des trois vallées avec celles de la Tanghène et de la Tipindjé, trente cinq propriétaires " Blancs ", disposant pour la plupart d'un autre revenu dans la fonction publique ou le commerce, se partageaient 6500 hectares dont 380 de caféières. Mais, en tenant compte des parcelles confiées en métayage à d'anciens travailleurs au contrat, recrutés avant-guerre à Java, et à quelques rares Mélanésiens, le nombre d'exploitations agricoles hors " réserves indigènes " s'élevait à 66 .
Des fragilités lézardaient cet édifice : le vieillissement de sa population coloniale, l'absentéisme affectant la grande propriété (qui couvrait la moitié des surfaces concédées) et le manque de main-d'œuvre pour la récolte du café. On pouvait en déduire que cette emprise foncière d'une poignée de colons serait difficilement tenable face à la lente mais régulière croissance démographique mélanésienne et à l'amenuisement des réserves foncières domaniales susceptibles de venir agrandir des " réserves indigènes» devenues insuffisantes.

Quelques propriétaires européens, en prenant de l'âge, songeaient à se débarrasser de leur concession. Dans la haute Hienghène, on évoquait la prochaine mise en vente d'un vaste domaine d'élevage tardivement constitué par additions de nouvelles concessions entre 1926 et 1961, la station Castex. Cette perspective suffisait à réveiller la mémoire mélanésienne et à raviver le désir de récupérer ces anciennes terres perdues au tournant du $\mathrm{XIX}^{\mathrm{e}}$ et $\mathrm{du} \mathrm{XX}^{\mathrm{e}}$ siècle. La chefferie Goa réclamait le retour de ces terres sous forme d'un agrandissement de la réserve. 


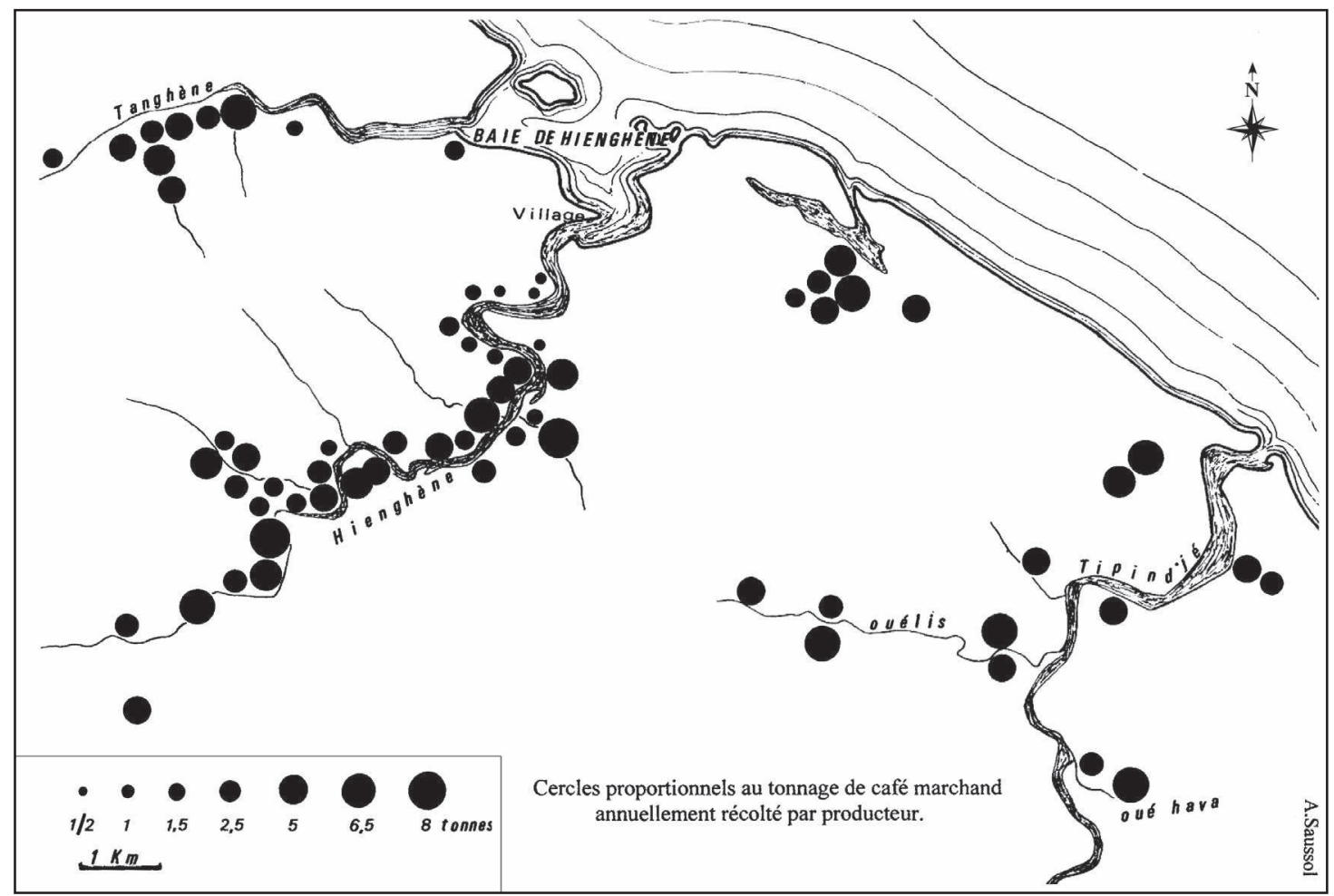

FIgure 3. - La caféiculture de colonisation à Hienghène à la fin de la décennie 1960

Les cercles indiquent les productions de café marchand par colon et par métayer dans les trois vallées. La circonscription de Hienghène produit alors en moyenne 240 tonnes de café marchand par an (95\% en Robusta). Dans ce total, la contribution des Kanak (non figurée sur la carte), reste faible. Les Kanak manquent d'espace et doivent partager les terres cultivables peu étendues de leur " réserve» entre petites plantations de café et champs vivriers.

Mais les gens de sa tribu en demandaient le partage sous forme de lots constituant autant de petites propriétés individuelles où ils seraient plus indépendants. L'Administration entendait donner satisfaction aux deux parties. Elle voulait se concilier le grand chef mais, en même temps, le principe de la "réserve indigène " présumée collective lui semblait inefficace. Elle y voyait un archaïsme freinant l'intégration des Mélanésiens dans le monde moderne et l'idée tendait à prévaloir qu'il valait mieux faire accéder les Kanak à la propriété individuelle assortie de mesures protectrices. Tel était l'un des débats de ce temps.

L'autre enjeu était le maintien de la caféiculture. En apparence celle-ci était triomphante. La moyenne vallée de la Hienghène, sous les frondaisons de ses arbres d'abri, était une coulée continue de café Robusta. La piste s'y enfonçait dans la pénombre des bois noirs et des érythrines qui estompait les vieilles maisons coloniales et engloutissait les cases plus modestes des métayers, fermant toute échappée visuelle vers la rivière ou la montagne.

La production avait retrouvé en 1950 son niveau d'avant-guerre. Depuis, elle stagnait. Les Européens s'en désintéressaient. Pour pallier le manque de main-d'œuvre, le recours au mé- tayage indonésien s'était généralisé. On avait même commencé à installer des métayers mélanésiens. Or, sauver la caféiculture, grevée par le coût croissant du travail, passait par l'élimination de ce métayage qui, en amputant d'un tiers des revenus déjà faibles, s'avérait dissuasif. La solution qui semblait s'imposer était le rachat d'une partie des caféières des colons et leur transfert aux métayers pour créer un paysannat de petits propriétaires asiatiques ou autochtones. Mais pour ne pas créer de trop grandes disparités et prendre en compte la " coutume » il était nécessaire d'en attribuer une autre partie, à titre collectif, aux Kanak demeurés dans les réserves qui étaient de loin les plus nombreux. Toutefois, pour rendre ces transferts acceptables aux Européens peu enclins au partage, on pouvait imaginer que ceuxci, arrivés à l'âge de la retraite ou leurs fils partis s'embaucher hors de la vallée, conserveraient leur maison avec une parcelle de terrain comme patrimoine familial. Tel était le schéma voulu pluri-ethnique vers lequel il semblait raisonnable d'aller, un retour exclusif de toutes les terres de la vallée aux Kanak étant alors inimaginable.

Quant au village de Hienghène, il était encore tel qu’au début du siècle. Au débarqué du bac, en prenant la route terreuse qui le traversait, on passait devant un comptoir de brousse tenu par 


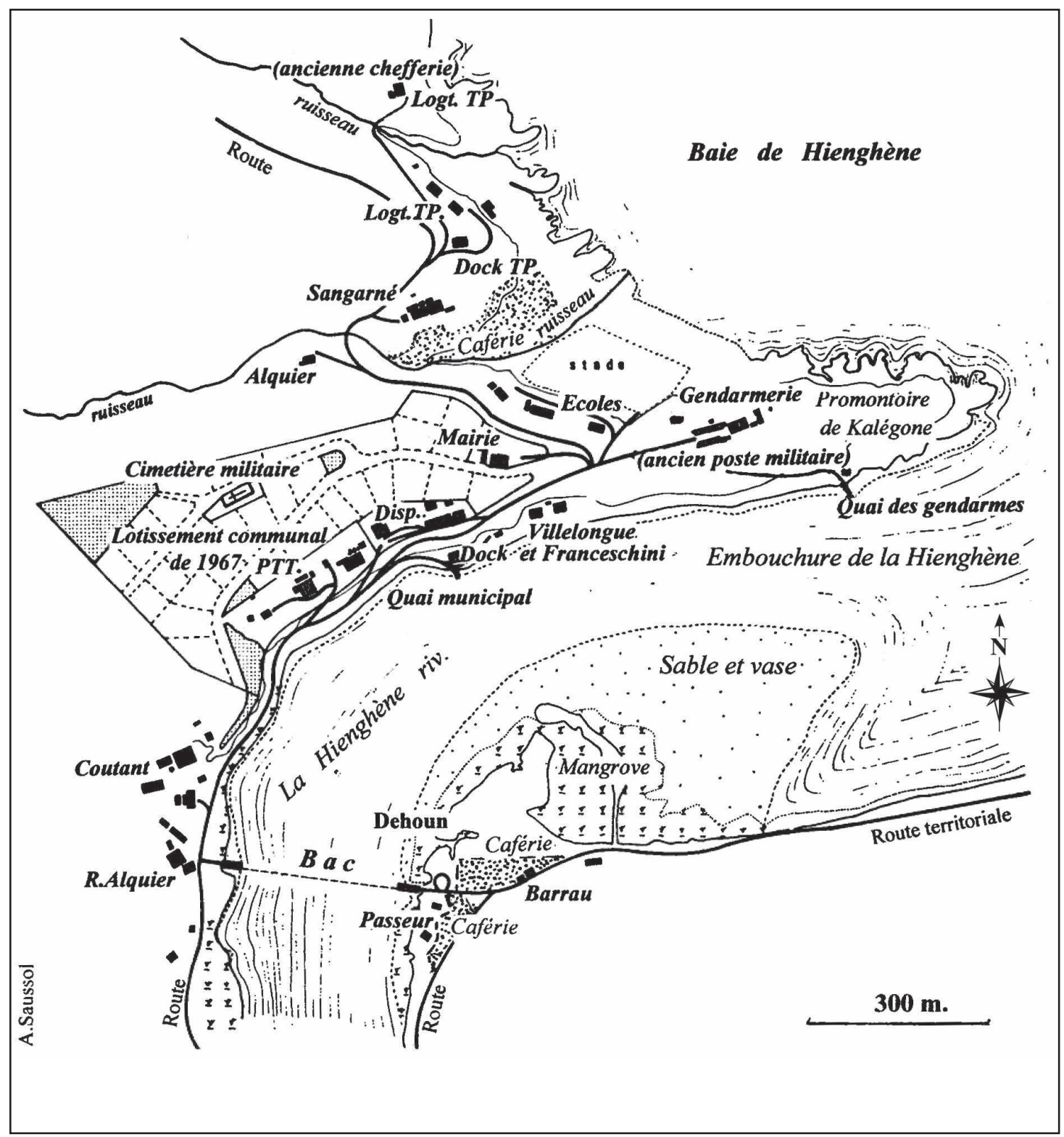

Figure 4. - Village de Hienghène en 1967 (d'après les travaux des géomètres Lethézer et Beyney)

Plutôt qu'un village, un centre réduit à sa plus simple expression alignant des services de première nécessité pour une population installée sur les concessions ou dans les réserves.

un Européen, une petite " usine à café ", plus loin l'agence postale, puis le dispensaire avec logements attenants. En contrebas de la piste, au bord de la rivière, une épicerie faisait office de débit de boisson. La traversée culminait au promontoire de Kalégone entre la mairie accrochée au versant et la gendarmerie logée dans le vieux fort construit en 1880 sur la presqu'île. Sous le rempart de cet ancien poste militaire, non loin du rivage, l'école avec les logements des instituteurs. Au-delà, encore une ou deux habitations éparses, l'entrepôt des Travaux publics flanqué des logements de ses agents, avant que la brousse ne reprenne possession du versant. On pouvait dépasser Hienghène et atteindre la mission de Ouaré où régnait encore la figure emblématique du vieux père Rouel, sans avoir réalisé qu'on venait de traverser un village. Les habitants résidaient dans les vallées, chacun chez soi : colons et Javanais dispersés sur les concessions, Mélanésiens groupés en petits villages dans leurs réserves. À quinze kilomètres à l'est de Hienghène, vers Koulnoué, au bord d'un petit terrain d'aviation plus que rustique, fraîchement dégagé dans la cocoteraie littorale, naissait un modeste "faré » touristique appelé à connaître un grand développement. Pour l'heure, endormie au bord de sa baie, face au rocher Pura qu'on appelait encore les "Tours de Notre-Dame ", Hienghène semblait vivre hors du temps. Le choc des réalités n'allait plus tarder à le ramener dans le siècle. 


\section{La montée des revendications}

\section{Une décennie de confrontations (1975-1985)}

Depuis 1940, pendant une génération, les structures foncières et démographiques du pays de Hienghène étaient restées stables. Dans l'immobilisme ambiant, les dernières pressions du front de colonisation en direction de la haute vallée avaient fini par s'éteindre. Entre 1965 et 1970, la situation est figée. C'est alors qu'un "boom " économique fondé sur d'ambitieux projets d'extraction du nickel, secoue la Brousse rurale. Les jeunes de toutes ethnies partent vers le mirage des mines et de Nouméa où les banlieues commencent à proliférer. Trois ans plus tard, la récession et le chômage ramènent les jeunes Kanak frottés de vie urbaine mais déçus, vers leurs réserves natales où la terre manque pour les accueillir. N'y trouvant plus leur place, ils en veulent aux «Blancs " détenteurs de l'essentiel des terres et des emplois locaux. Et, n'ayant plus peur d'exprimer leur ressentiment, ils le disent. La jeune génération mélanésienne commence à se confronter à la colonisation vieillissante. Le thème de la reconquête des terres perdues devient récurrent. Des " litiges fonciers " opposant colons et Kanak pour la possession du sol se multiplient le long de la côte Est. Ces derniers réclament désormais « la reconnaissance de droits particuliers pour leurs collectivités ».

Au cours des années suivantes, partout dans l'île, la situation se durcit et se politise avec la création de mouvements kanak. Les tensions s'exacerbent autour des litiges fonciers. Jeunes Européens et Javanais, inquiets, quittent la brousse. Voulant calmer le jeu et prévenir un glissement politique de ce qu'il pense être encore un problème foncier, le gouvernement de Giscard d'Estaing concocte un plan de développement appuyé sur la création d'un fonds spécial et sur le projet d'une réforme foncière lancée en 1978.

Cette dernière débute par un inventaire des terres revendiquées, qui réveille tout un passé de frustrations et révèle l'ampleur de la revendication. À Hienghène, les gendarmes, chargés de l'enquête, doivent dresser la carte des terres dont le retour est réclamé par les clans. Ils passent donc chez les colons. Ceux-ci, d'abord stupéfaits puis incrédules, se persuadent qu'on veut les chasser de chez eux. D'où leur indignation. En face aussi l'impatience grandit et, dès novembre 1978, l'ethnologue J. Guiart, constatant un climat extrêmement tendu à Hienghène, prévoit avec lucidité " un militantisme grandissant visant à rendre la situation intenable aux Européens ", malgré l'influence modératrice du leader local, Jean-Marie Tjibaou (récemment élu maire et vice-président du parti autonomiste Union calédonienne).
Dans le cadre de sa réforme foncière, le secrétaire d'État Paul Dijoud avait reconnu à chaque clan le droit légitime à retrouver la terre de ses ancêtres. Élargissant le champ d'application de cette logique, l'ensemble des clans de Calédonie défini comme " peuple kanak " représenté par ses élus, allait réclamer la souveraineté sur l'ensemble de l'archipel, rebaptisé Kanaky. En s'élargissant du particulier au général, de foncière la revendication devenait politique.

En trois ans, entre 1976 et 1979, l'idée d'indépendance fait son chemin. Même l'Union calédonienne, vieux parti autonomiste construit sur le slogan " deux couleurs un seul peuple ", finit par s'y rallier. Aux élections territoriales de 1979 se constitue un Front indépendantiste avec pour mot d'ordre : « indépendance kanak socialiste ». Par contrecoup, dans un climat d'inquiétude et d'agitation croissante, les Européens rallient massivement le parti conservateur gaulliste-loyaliste (RPCR). Désormais, partis « caldoches" et " kanak " vont se structurer et s'affronter autour de cette question de l'indépendance.

En 1981, en France, l'arrivée des socialistes au pouvoir est une divine surprise pour les indépendantistes mélanésiens dont le projet semble soudain à portée de main. Mais Mitterrand entend gagner du temps et veut poursuivre la réforme foncière dont il escompte une meilleure intégration des Mélanésiens dans la vie économique. Pour les militants kanak, c'est une amère déception.

Dans cette ambiance de désenchantement, quatre mois après l'élection présidentielle, l'assassinat, près de Nouméa, dans des circonstances mal élucidées, du secrétaire général de l'Union calédonienne détériore gravement le climat politique local. Une phase d'extrême tension s'ensuit avec un début de mouvement insurrectionnel à Canala où émerge la figure charismatique d'Éloi Machoro qui appelle à l'épreuve de force contre les «Blancs », invitant les «tribus » à « dresser la liste de ceux qui devront partir » et proclamant sa détermination "à tirer s'il le faut ". Coup de semonce de la révolte en gestation. Pour calmer les esprits et contrôler la situation, l'État central se substitue au pouvoir Territorial et entreprend de gouverner par ordonnances. La création d'un Office foncier doit lui donner les moyens d'agir efficacement.

À cette date, la réforme foncière à Hienghène n'a eu encore aucun effet concret. Le sentiment prévaut que rien ne bouge. On attend. La commune compte alors 1528 Kanak et 150 Européens. Les Indonésiens, évaporés lors du boom du nickel, ne sont pas revenus malgré la récession et ne sont plus que 27. À la place des trois groupes ethniques antérieurs coexistant dans un paternalisme plus ou moins débonnaire, la vallée glisse, dans un climat tendu, vers une confrontation binaire Kanak/Caldoches lourde de menaces et d'arrière-pensées. 


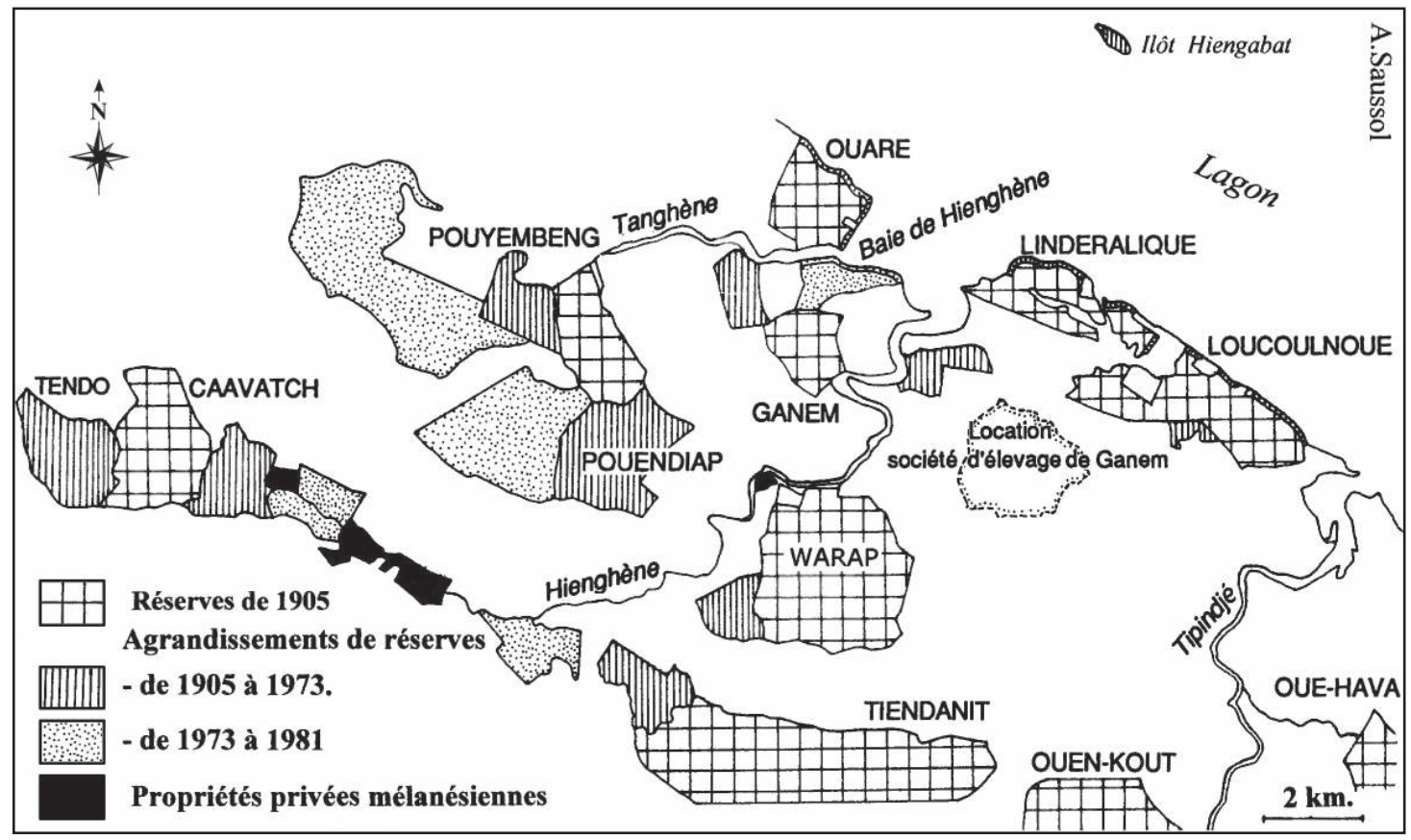

Figure 5. - Les terres détenues par les Kanak à Hienghène en 1981 (avant l'invention du GDPL)

Si les réserves, plusieurs fois agrandies depuis 1945, représentent l'essentiel des terres kanak, il existe aussi quelques petites propriétés privées appartenant à des Mélanésiens (notamment dans la haute Hienghène sur l'ancienne station Castex). Elles résultaient des efforts de l'Administration, vers 1970, pour mettre en place, en dehors des réserves " collectives » jugées archaïques et sclérosantes, une paysannerie mélanésienne moderne, de type occidental. Cette expérience sera abandonnée avec la montée des revendications foncières mélanésiennes, au nom du retour aux terres ancestrales et aux systèmes coutumiers.

Malgré l'Office foncier, le contexte calédonien continue de se radicaliser. Pour hâter la récupération et la redistribution des terrains, de bruyantes occupations de terres se succèdent. Renouant avec une ancienne coutume, les Mélanésiens dressent sur les propriétés des colons des cases symboliques affichant leur revendication. Devant la multiplication de ces constructions, au début de 1984, l'Office (qui n'a pas les moyens de garantir les droits des nouveaux propriétaires) se trouve débordé puis discrédité. Il va au plus pressé, transférant les terres sous forme d'agrandissements de réserves ou de "groupements d'intérêt économique " que les désaccords entre clans paralysent, entraînant de nombreux échecs. En guise de développement, le lopin vivrier ouvert dans la friche ou le petit troupeau divaguant dans la brousse constituent les formes les plus habituelles de mise en valeur des terres restituées. En termes de développement la réforme est un échec. Mais l'enjeu n'est plus là. Voici longtemps que la revendication politique d'indépendance a éclipsé la simple réclamation des terres perdues. Lancien " Front indépendantiste " s'est mué en « Front de libération Kanak et socialiste" (FLNKS). Tout un programme.

Les idées aussi ont cheminé. En juillet 1983, à Nainville-les-Roches, « loyalistes " " caldoches "
(Européens nés en Calédonie) et «indépendantistes " kanak se sont mis d'accord (du bout des lèvres) sur le principe de l'abolition du fait colonial en Nouvelle-Calédonie et sur la reconnaissance de la légitimité du peuple kanak. Ils ont aussi accepté le principe d'une auto-détermination. Mais la question épineuse est devenue la définition du corps électoral. Qui, au bout du compte, doit être appelé à voter ?

Sur cet enjeu capital, les Kanak entrent en désaccord avec le projet de statut préparé par le secrétaire d'État Lemoine. Du coup, la tension remonte. En avril 1984, Lemoine venu "vendre " sa réforme à Hienghène est gratifié d'un accueil glacial. Il s'entend répliquer au nom des chefs coutumiers, habituellement modérés :

«Après avoir tant espéré [...], nous allons prendre notre destin en main. Des revendications de terres ont commencé et elles s'arrêteront le jour de l'indépendance de notre pays. "

C'est une fin de non recevoir. En novembre suivant, le FLNKS, nouvellement créé avec tous les partis kanak indépendantistes, appelle au «boycott actif» des élections territoriales. Elles doivent avoir lieu le 18 novembre 1984. La mobilisation mélanésienne s'organise. Le jour du scrutin, à travers tout l'archipel, la brousse 
entre en quasi-insurrection : insultes et menaces contre les votants, bris d'urnes, contrôle des routes, barrages, " caillassages" (jets de pierre sur les véhicules), incendies d'habitations, laissent le pouvoir d'État désemparé, muet.

\section{Le massacre de Wanyatt (5 décembre 1984)}

À Hienghène, deux semaines durant, les jeunes Kanak, transcendés par l'action, ont pris possession du territoire sans ménagement. L'Etat de droit s'est effondré, abandonnant les citoyens à leur sort face aux événements. Européens, presque tous armés, et gendarmes (auxquels l'autorité a retiré leurs armes avec ordre de ne pas résister), s'enferment chez eux ou se groupent au village. Le soir, des maisons désertées de colons brûlent, ancienne pratique de guerre visant ici à empêcher le retour des occupants. Dans ces convulsions où agonise un siècle de colonisation, des rumeurs alarmistes courent. Menaces de désarmer les « Blancs " d'un côté, bruits d'une intervention imminente des "milices à Lafleur" pour les secourir, de l'autre. Cette ambiance crépusculaire s'éternise. Au soir du 5 décembre 1984, une poignée de petits colons craque et commet l'irréparable. Sur la route qui remonte la vallée de la Hienghène, ils tendent une embuscade aux membres du " comité de lutte » regagnant leur tribu en camion après une réunion au centre culturel. Grinçante ironie, il venait, diton, d'y être question de lever les barrages et de suspendre les actions. Trop tard. Dans la nuit de Wanyatt, la fusillade crépite. C'est un massacre. Dix jeunes de la tribu de Tiendanite restent sur le terrain ; parmi eux deux frères du leader mélanésien Jean-Marie Tjibaou.

Pour les Européens de Hienghène, commence alors le dernier acte de leur tragédie. Les meurtriers ont aussitôt fui dans la Chaîne centrale pour s'y cacher. Quelques jours plus tard, ils se rendront aux autorités. A l'issue du procès, ils se verront acquittés et acclamés à Nouméa, naufragés pathétiques de l'histoire devenus les héros pitoyables d'un soir, sitôt oubliés, au prix de leur vie dévastée. Affolés par la peur de représailles, les autres non-Mélanésiens de la vallée se réfugient à la gendarmerie d'où on les évacue en catastrophe. Entre le 4 et le 6 décembre, près de 300 d'entre eux (venus de toute la région) partent pour un exode définitif. Ils ne reviendront pas et devront se résoudre à vendre leurs terres à l'ADRAF (Agence de développement rural et d'aménagement foncier) qui les restituera aux Kanak sous forme de groupements dits " GDPL ».

L'intermède colonial s'est sèchement dénoué à Hienghène dans le drame de Wanyatt. Les Kanak, vainqueurs de la confrontation, y sont désormais seuls. Dès lors se pose la question du devenir des terres reprises et de la manière dont elles vont être réparties et gérées. Irait-on, comme le voudraient certains, vers une tentative de reconstitution utopique de l'ancien pays kanak précolonial ? Ou bien, comme l'imaginent d'autres, à partir d'une table rase, vers la construction d'un système nouveau d'inspiration socialisante ? Y répondre revient à constater ce qu' est devenu Hienghène depuis cette date. À défaut d'une enquête approfondie, les observations faites sur le terrain et les discussions avec différents acteurs permettent d'apporter quelques éléments de réponse.

\section{Hienghène aujourd'hui}

\section{Le pays repeuplé}

Depuis 1984, la population de Hienghène a profondément changé, d'abord avec la disparition des Européens (et des Javanais), réduits aujourd'hui à quelques fonctionnaires, pour la plupart sans attaches locales, et aux gérants du complexe touristique de Koulnoué. Pourtant, Hienghène n'a jamais été aussi peuplé. C'est le résultat d'une forte augmentation de la population mélanésienne qui a connu une soudaine accélération entre 1984 et 1989 avant de se poursuivre à un rythme plus modéré mais toujours soutenu après 1990. Résultat : entre 1969 et 2004 , le nombre de Kanak à Hienghène a cru de $80 \%$, passant de 1420 à 2 538. Ce quasidoublement se perçoit physiquement jusque le long du littoral où des sites jadis déserts sont aujourd'hui occupés. Le pays vit. Les routes (toutes goudronnées) sont plus animées, la circulation automobile est bien plus intense qu'autrefois.

\section{La fin du café}

Dans les années soixante, Hienghène était un gros centre de caféiculture. Ce n'est plus le cas aujourd'hui. À partir de 1964, minée par un parasite, le "scolyte" (Stephanoderes hampei), la production de café s'affaisse. Elle était alors de 223 tonnes par campagne (moyenne triennale 1964-1966). Au début des années 1970, lors $\mathrm{du}$ " boom " économique, alors que se propagent les "fourmis électriques ", elle est tombée à 135 tonnes (moyenne triennale 1970-1972). La lutte anti-scolyte la stabilise à ce niveau (132 tonnes, moyenne triennale 1977-1979) sans que les motifs d'inquiétude quant à son avenir aient disparu. Au milieu des années soixante, on avait envisagé une relance de grande envergure, avec l'implantation d'une station expérimentale de l'Institut français du café et du cacao (IFCC) à Ponérihouen. Là sont effectués des essais de caféiers sans ombrage (localement appelés « caféssoleil ") et sont mises au point les interventions agronomiques, phyto-sanitaires ou de traitement 


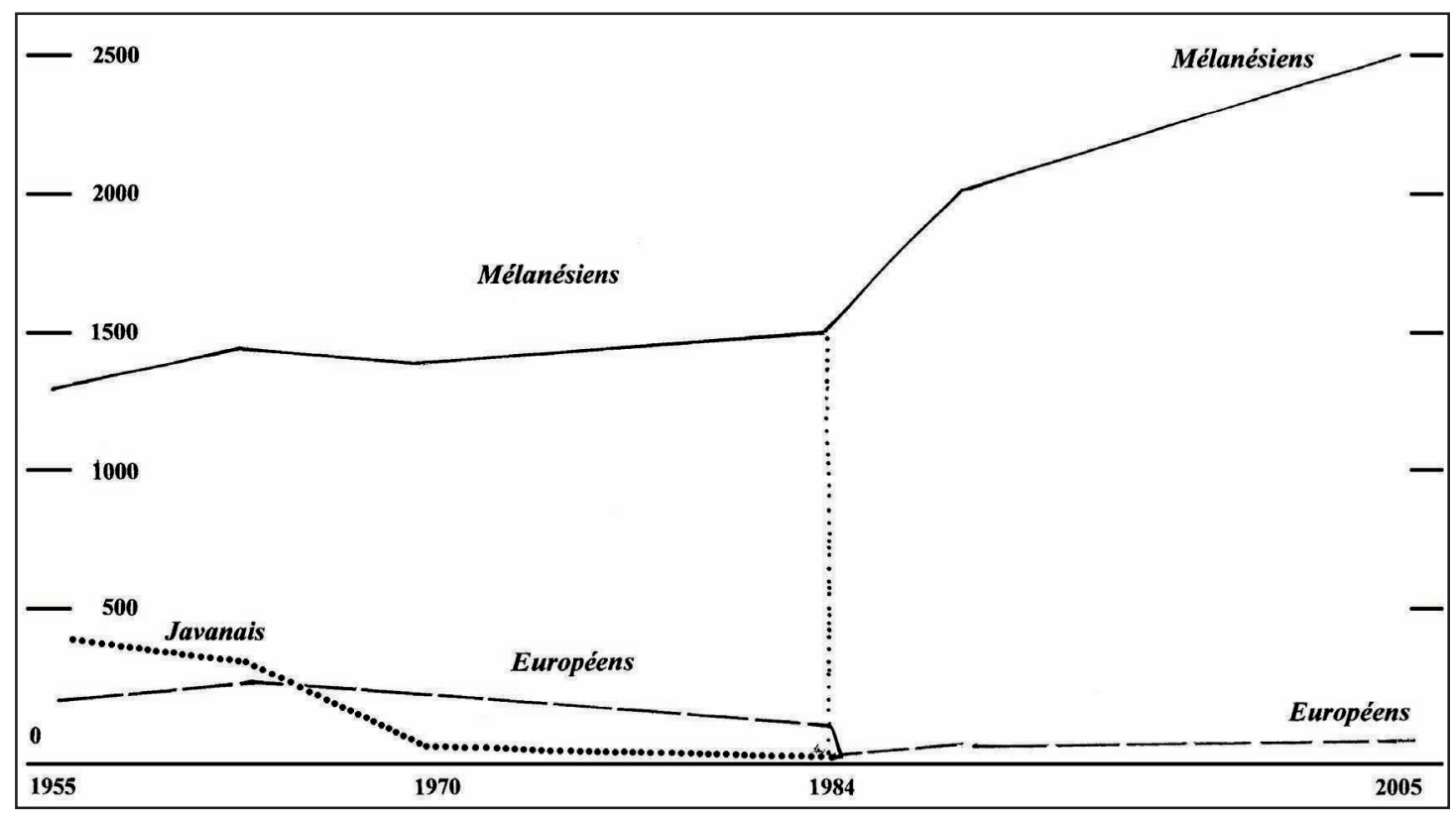

Figure 6. - Évolution de la population de la circonscription de Hienghène entre 1955 et 2005

Des trois groupes ethniques qui cohabitaient dans les années cinquante, seul subsiste aujourd'hui celui des Kanak. Les Javanais, plus nombreux que les colons au lendemain de la Deuxième Guerre mondiale, ont disparu en tant que groupe, lors du brusque appel de main-d'œuvre suscité par le boom économique de 1970. Ils ont alors abandonné leurs métairies caféicoles pour aller occuper des emplois salariés dans le Sud. Les colons vieillissants, affaiblis par l'exode des jeunes Européens, déclinaient depuis la fin des années soixante. Le massacre de Wanyat (fin 1984) leur porte un coup fatal. Les Mélanésiens, ayant repris l'ensemble de leurs terres ancestrales, connaissent, depuis cette époque, un renouveau démographique. Quant aux Européens établis aujourd'hui à Hienghène, ils n’ont rien à voir avec la vieille colonisation rurale : ce sont de nouveaux venus, souvent métropolitains, arrivés après 1984 comme fonctionnaires ou employés dans le secteur touristique.

des cerises par voie humide, destinées à améliorer la qualité des grains et à relancer la production. Après ce premier train d'expérimentations et d'interventions, sera lancée en 1980 une ambitieuse "Opération café » visant à créer 2000 hectares de caféières modernes pour faire passer, en dix ans, la production calédonienne de café marchand, de 600 à 3000 tonnes. Espoir déçu. À partir de 1983, la Nouvelle-Calédonie cesse d'exporter du café et en importe chaque année davantage. En 1990, le tiers des nouvelles parcelles implantées est à l'abandon. Deux ans plus tard, l' " opération café » est arrêtée sur un constat d'échec.

Les moyennes triennales de production de café marchand sont passées de 400 tonnes en 1981 1983 à 268 tonnes pour 1987-1989 ; puis à 94 tonnes pour 1990-1992, et à une trentaine de tonnes pour 2000-2002 comme pour 20062008. Le pays de Hienghène s'inscrit dans ce mouvement. Comme partout, les raisons du déclin sont multiples et interdépendantes. Désintérêt pour une culture symbolisant l'ordre colonial sous ses deux formes, brutale puis paternaliste ? Pas sûr. Au niveau des "représentations ", nombre de Kanak se disent attachés au café en lequel ils voient un moyen d'accès commode et autonome à des revenus réguliers et rechignent à arracher leurs plantations. Mais d'autres raisons plus terre à terre se cumulent pour expliquer les abandons. En vrac:

- les dégâts du scolyte qui trouve dans les plantations abandonnées des foyers de reproduction idéaux ;

- les irritations insupportables provoquées par les piqûres des "fourmis électriques " devenues le fléau des récoltes ;

- des contraintes imprévisibles comme les aléas climatiques ou les troubles politiques ;

- la réduction par le Groupement agricole des producteurs de la côte Est (GAPCE) du nombre de ses points de collecte et notamment la fermeture de celui de Hienghène ;

- la difficulté matérielle d'effectuer la récolte lors de la courte période de maturité des cerises ;

- le prix de la main-d'œuvre ;

- la longueur des délais de paiement, pouvant atteindre une année ;

- la difficulté de créer de nouvelles plantations de plein soleil exigeant un gros effort sans rémunération immédiate car ne devant produire qu'au bout de trois ans.

Dans ces conditions, les gains apportés par le café aux producteurs restent largement inférieurs au revenu minimal calédonien. S'ajoute à tout ceci la concurrence des cultures "vivrières ", de plus en plus commercialisées. 
Pour toutes ces raisons, le café s'est effondré à Hienghène. En 1991, à la veille de l'abandon de l' "Opération café ", sur 65 hectares de caféières dénombrés sur la commune de Hienghène, 42,5 ha étaient exploités parmi lesquels 33 seulement étaient entretenus (la moitié de l'ensemble, l'autre moitié étant à l'abandon). Après cette date, le potentiel de production estimé de ces caféières a dégringolé de 60 tonnes en 1992 à 34 tonnes en 1995. La décennie 1990 s'avéra catastrophique. En 2001 et 2002, il n'y a pas eu de production à Hienghène, ou si faible qu'elle n’a pas été comptabilisée. En 2003 et en 2004, la récolte fut insignifiante (respectivement 500 et $2350 \mathrm{~kg}$ ). Depuis 2005 , Hienghène n'a plus livré de " cerises " au GAPCE. Le temps du café y semble terminé.

La transfiguration paysagère qu'induit cette mutation saisit quiconque revoit la vallée une génération après l'avoir connue. À la place de l'ancienne coulée de caféières dans la pénombre des ombrages, alternent des paysages plus dégagés et plus ensoleillés : champs de cultures vivrières, parcelles embroussaillées, plantations à l'abandon ou mal entretenues parmi lesquelles parfois quelques parcelles de "café-soleil ». Du coup le paysage de la vallée, jadis étouffé par la densité du couvert végétal, se dégage et réapparaît. Au confluent de la Pouendiap, dressé au-dessus de la rivière, on voit désormais surgir le promontoire de Kamédane, ancien site de la chefferie Bouarate d'accès interdit mais redevenu visible.

Les cultures vivrières en lopins discontinus n'occupant qu'une petite partie de l'espace sont devenues la principale production du pays. À leur ancienne fonction autarcique, elles ajoutent une ouverture vers un marché local étroit, limité au village et aux clients de l'hôtel de Koulnoué devenu un gros complexe touristique de niveau international, mais aussi vers des consommateurs plus lointains grâce au colportage. La plupart de ces parcelles sont cultivées par des membres du groupe propriétaire. Quelques-unes sont louées à des gens du pays (pas nécessairement mélanésiens) pour être mises en valeur. Ce retour aux tubercules ancestraux (tel que l'igname qui se vend bien) ou à la production légumière, face à l'effacement de la culture de traite caféicole, suggère une adaptation moderne de l'ancienne tradition agricole mélanésienne refermant la parenthèse coloniale.

\section{La réoccupation des terres}

Entre 1967 et le début des années 1980, malgré les tensions politiques et la montée des revendications, à Hienghène, les terres détenues par les Européens n'avaient guère bougé hormis le rachat par le territoire d'une station d'élevage (Castex) ou de quelques concessions éparses, et le déclassement, en bord de mer, de portions de zone maritime. Vers 1975, ces terres avaient été données en agrandissement aux " réserves" voisines ou, dans la haute Hienghène, concédées en petits lots de propriété individuelle de 3 à 5 hectares à des Kanak, des Javanais, et même à quelques Européens de la vallée. Dans la Tipindjé, rien n'avait changé. À cette époque, $41 \%$ des terres détenues par les colons de Hienghène l'étaient par des gens âgés de plus de 65 ans. De ce fait, 4700 hectares étaient susceptibles de se libérer assez vite. En attendant, rien ne bougeait : la réforme foncière s'enlisait face à des revendications à rallonge. Étonnant immobilisme, alors que les tensions étaient vives. Les Européens de Hienghène ne semblaient pas en avoir pris conscience jusqu'à ce qu'en décembre 1981, la colère éclate avec une violence inattendue à Canala. À partir de là et jusqu'en 1984 s'ouvre une période d'exaspération pré-insurrectionnelle qui va finir par embraser l'archipel.

Dans les années qui suivent le massacre de Wanyatt, Hienghène reste assommé sous le choc. Le village est mort. La vie s'y fige dans la stupeur et le deuil. Peu à peu pourtant, elle reprend sur des fondements différents. Dès 1985, les colons expulsés commencent à vendre leurs terres abandonnées. Celles-ci devaient être rétrocédées aux clans coutumièrement propriétaires. Mais, reposant sur une tradition orale altérée par le temps, confrontée à des stratégies lignagères antagonistes, l'identification des propriétaires coutumiers s'avérait longue et parfois problématique. Le temps passait en discussions et en palabres où les gens s'épuisaient sans voir aboutir leurs revendications. Il importait d'en sortir. On s'interrogeait aussi sur la façon de concilier possession coutumière du sol et contraintes imposées pour une mise en valeur spéculative, deux concepts paraissant incompatibles.

\section{La formule du "groupement de droit particulier local» (GDPL)}

Au début, on avait esquivé la question en restituant les terres sous forme d'extensions des réserves et en laissant les Kanak se débrouiller pour les répartir entre eux. Mais, en 1982, pour permettre à la propriété coutumière de répondre aux contraintes de l'économie de marché, l'Office foncier avait inventé le GDPL, censé prendre en compte la coutume et doté de la personnalité morale et juridique.

Il s'agit d'une structure juridique pragmatique, propre à la Nouvelle-Calédonie, facile à créer, constituée par des membres représentés par leur mandataire. Son substrat foncier forme une sorte de propriété sociétaire, inaliénable, insaisissable, gérée en interne selon les modalités coutumières et, en externe, d'après les règles de l'économie dominante. Le GDPL devient le nouveau propriétaire foncier. Passage obligé pour les attributions de 


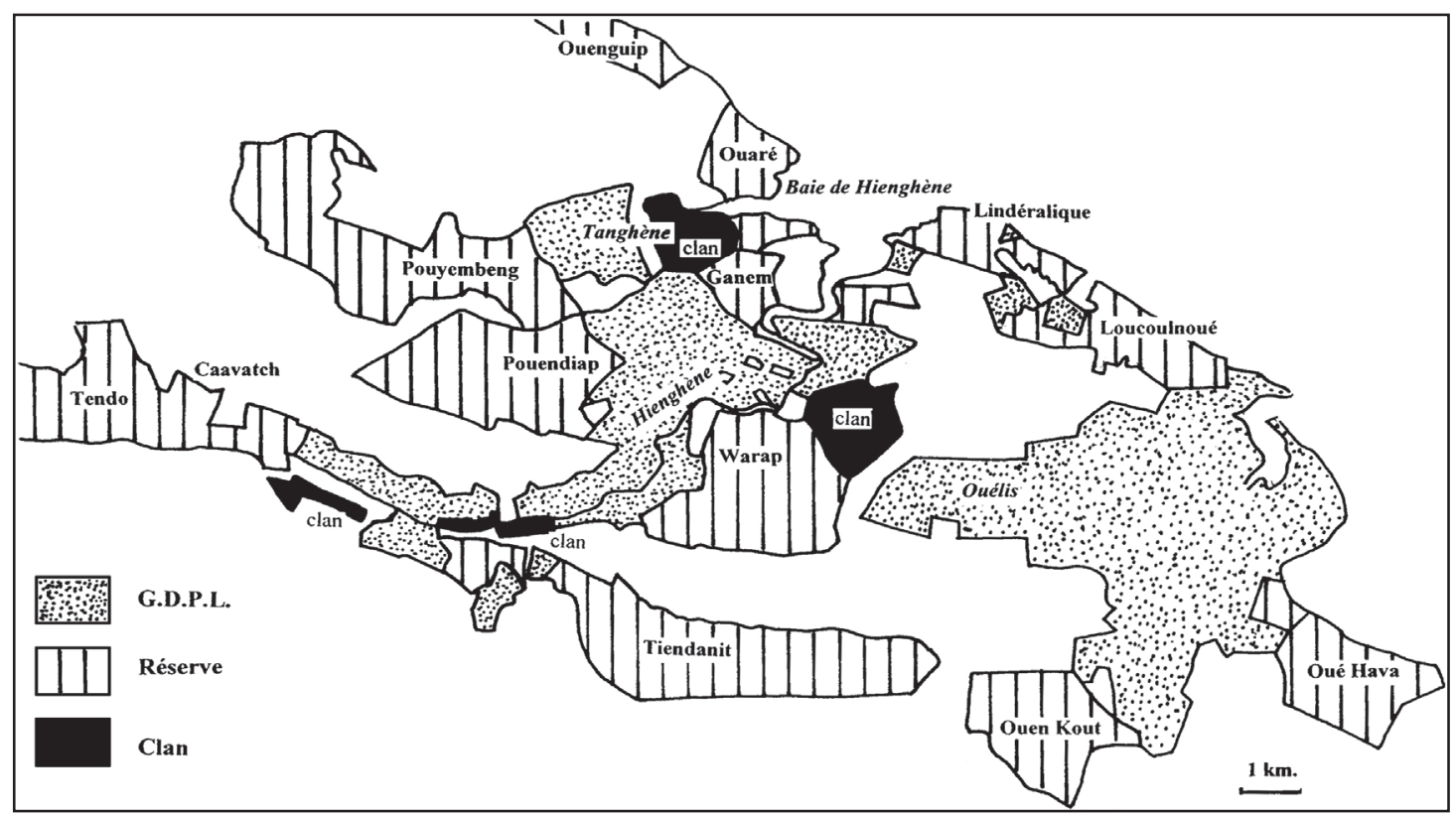

Figure 7. - Formes juridiques de réoccupation par les Kanak des terres de colonisation

Après 1984, les Kanak ont réoccupé les terres des anciens colons sous forme d'agrandissements de réserves, d'attributions directes à des clans ou, le plus fréquemment, de "groupements de droit particulier local » (GDPL). Les rares concessions privées, antérieurement accordées à quelques Mélanésiens, dénoncées par les indépendantistes comme un héritage colonial, ont disparu, absorbées par les nouvelles formes de contrôle du sol.

terres, il est une interface mais aussi un pont entre tradition et modernité. Il peut être clanique (voire polyclanique) ou tribal selon qu'il est attribué aux membres d'un seul (ou de quelques clans apparentés), ou à l'ensemble des habitants d'une « réserve » (formant une " tribu " en réunissant de nom- breux clans coutumièrement hétérogènes). Dans le second cas, jouxtant souvent la réserve, il tend à fonctionner comme un agrandissement de cette dernière mais enrichi des possibilités juridiques et financières ouvertes aux GDPL. Au bout du compte, grâce à ces groupements, la propriété coutumière

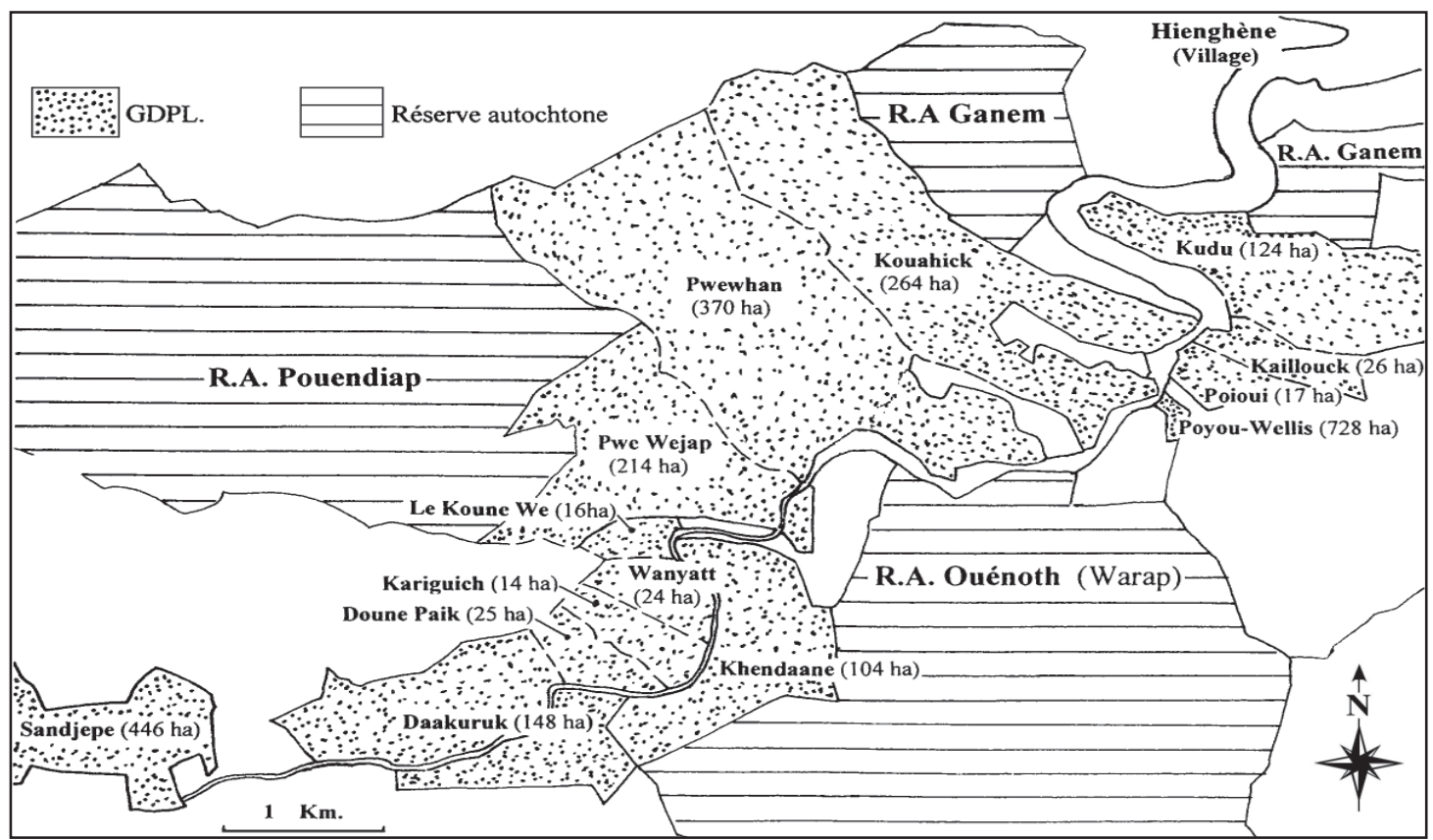

Figure 8. - Les GDPL dans la basse et la moyenne vallée de la Hienghène

Chaque GDPL a repris un nom ancien, oublié pendant la période coloniale. Leurs superficies (indiquées sur la carte) sont inégales. Ils peuvent inclure des terres localisées dans les vallées voisines. Ainsi le GDPL PouyouWellis a-t-il l'essentiel de ses 728 ha dans le bassin de la Tipindjé. 
a pu perdurer d'autant mieux qu'elle est devenue susceptible d'une mise en valeur spéculative.

Après 1995, les revendications non satisfaites sur d'anciennes terres coloniales s'étant multipliées, pour y répondre on recourt massivement aux GDPL qui deviennent l'instrument quasi-exclusif de la reconquête foncière. À Hienghène, presque tous les " groupements " datent de 1998. En 2004, la commune en comptait 31 couvrant 5059 hectares. Elle était celle qui en possédait le plus de toute la Grande Terre.

Les GDPL sont créés avec un périmètre précis et cartographié. Leurs limites épousent celles d'anciens lots de colonisation et se calquent donc sur d'anciennes concessions coloniales ou sur des portions de ces dernières. Cette inscription dans le cadastre colonial montre que les GDPL ne sont pas un décalque rigoureux des vieux territoires claniques précoloniaux. C'est au mieux une approximation, parfois une création entièrement nouvelle, notamment s'agissant de groupements " tribaux ". Il suffit alors que les maîtres coutumiers, s’ils existent encore, aient donné leur accord.

Il en va de même pour la localisation des nouveaux habitats. Avec la terre, les Kanak de Hienghène ont récupéré leurs anciens tertres claniques ou lignagers et ceux des chefferies. Toutefois, il serait illusoire de croire qu'on réoccupe systématiquement les sites des anciens hameaux abandonnés lors du cantonnement. Ceux-ci, souvent perchés sur le piedmont colluvial ou même à flanc de montagne, sont aujourd'hui inaccessibles faute de chemin. La brousse les a repris assurant la tranquillité des ancêtres. Les nouvelles implantations se font dans la vallée, en bord de route. Pas chez les anciens colons dont les habitations ont brûlé en 1984 et dont la brousse ensevelit les ruines, mais à l'écart, sur des emplacements nouveaux. Il s'agit d'un habitat contemporain conforme aux besoins de la société actuelle. S’il s'inscrit idéologiquement et affectivement dans la tradition et dans la continuité du vieux pays, le nouveau pays kanak, reconstruit après l'épisode colonial, n'est pas un décalque de l'ancien. Ce serait supposer que la géographie pré-européenne était figée alors qu'elle était dynamique. Aux temps anciens aussi les hommes bougeaient. L'espace vivait, se transformait, lentement mais en permanence. Des hameaux pouvaient être abandonnés et d'autres se créer ailleurs, jalonnant les itinéraires des lignées. Avec leurs premiers levés, en cartographiant cases et hameaux, les géomètres coloniaux purent croire saisir une réalité intemporelle, l'ordre éternel des champs. Il ne figeaient sur le papier qu'un instantané, un moment d'une longue histoire. Ainsi comprise, la nouvelle géographie mélanésienne de la vallée ne s'oppose pas à l'ancienne. Elle ne la reproduit pas mais la prolonge en l'adaptant aux réalités d'aujourd'hui. C'est une des leçons de cette expérience post-coloniale.

\section{Les formes de mise en valeur}

Beaucoup de GDPL ont réparti leurs terres entre les familles issues de leurs lignages. Celles-ci les utilisent comme bon leur semble mais n'ont qu'un droit d'usage, la terre restant propriété de l'ensemble du groupement. Mais ces membres de GDPL qui quittent les « réserves " pour venir $s$ 'installer sur les terres de leur groupement doivent les exploiter.

On peut cultiver la terre soi-même ou la louer à un tiers, même extérieur au GDPL, pour la réalisation d'un projet. Pour cela, l'ADrAF a défini des baux ruraux destinés aux projets agricoles ou pastoraux et d'autres destinés à la construction, permettant de bâtir de l'habitat locatif ou des gîtes ruraux sur les terres des GDPL. Il s'agissait de dépasser le stade du simple accord verbal (pratiqué auparavant) pour trouver un moyen d'individualiser les droits fonciers sans recourir à l'appropriation privée. En 2004, pour l'ensemble de la Nouvelle-Calédonie, le tiers de la superficie de GDPL était ainsi loué à des particuliers. C'est dire que la formule a plu.

Mais, à Hienghène comme ailleurs, la mise en valeur des terres reste ponctuelle, révélant la prégnance d'une économie domestique aux ambitions modestes, intégrant un peu d'élevage, parfois la chasse au cochon sauvage et au cerf, la pêche ou d'autres petits profits. La gestion des GDPL pourrait être plus dynamique. Les « mandataires ", élus sans limitation de durée, semblent désignés à vie. On se dispute parfois pour l'argent du groupement. Aussi, les membres sont-ils souvent enclins à s'en partager le terrain pour l'exploiter individuellement. On ne peut exclure qu'à long terme, les GDPL ne finissent par glisser vers des formes d'appropriation individuelle. Ils auraient, dans ce cas, joué un rôle de transition.

Pour l'heure, entre ses terrains embroussaillés, ses plantations à l'abandon et ses champs de cultures vivrières dont une part de la récolte est vendue, la vallée vit au ralenti. La longueur des délais de transmission de la terre des colons vers les Kanak l'explique en partie. Treize ans d'incertitudes entre le départ des colons (1984) et la création des GDPL (1998) ont favorisé l'embroussaillement et le mauvais état des caféières. Tout comme l'exode des jeunes peu intéressés par l'activité agricole et moins motivés que leurs pères. C'est aussi un choix de vie des vieux Mélanésiens, heureux d'avoir retrouvé leurs terres ancestrales et sachant se satisfaire d'un train de vie modeste mais paisible qui leur convient, à l'écart des tensions générées par une activité spé- 

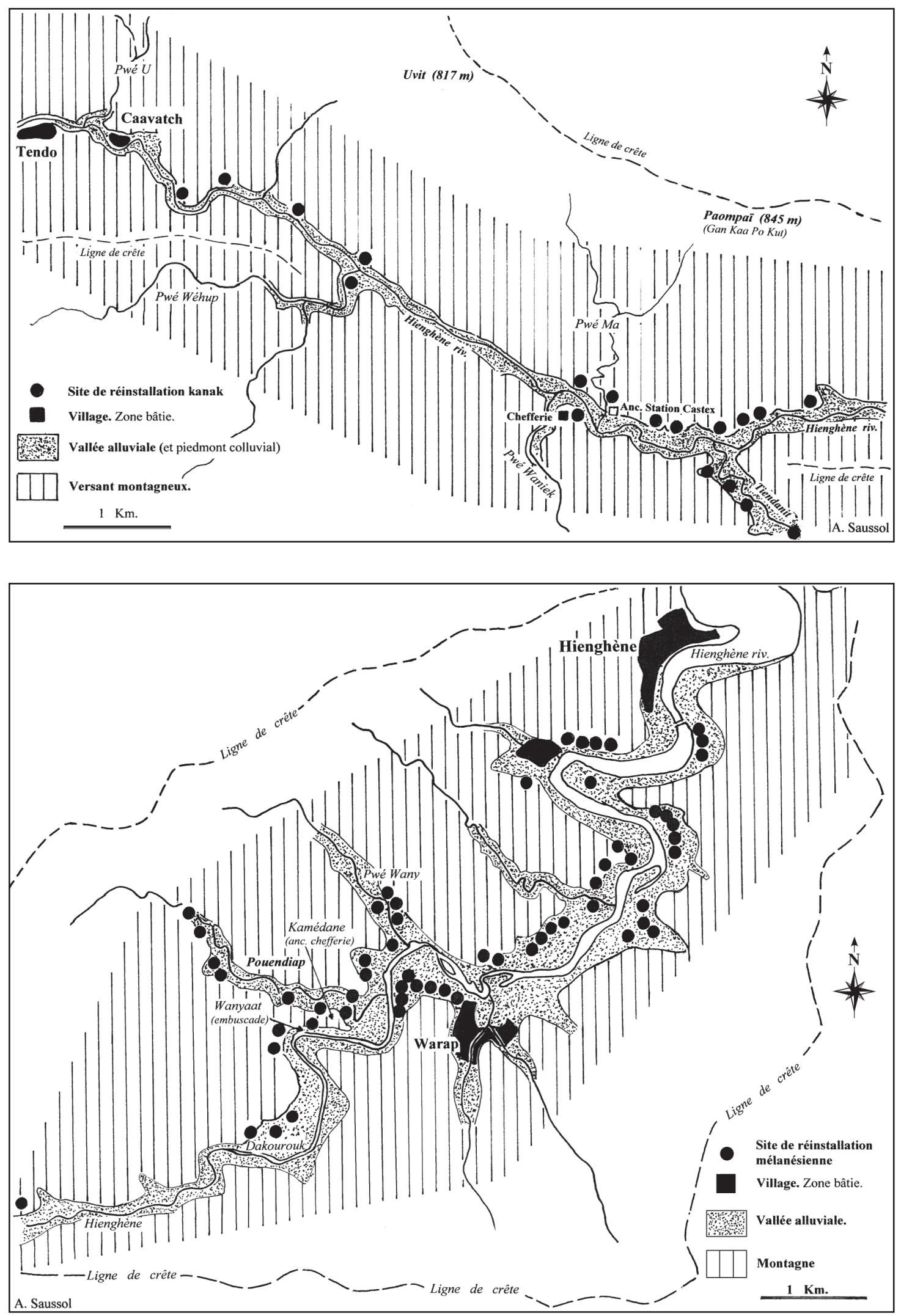

Figures 9-10. - Réoccupation de la vallée de Hienghène par les Kanak

Leurs nouveaux sites d'implantation hors des réserves, bien que tendant à retrouver la dispersion antérieure, ne correspondent pas exactement à ceux des hameaux précoloniaux. Leur localisation ne saurait être rigoureusement identique. Ils dessinent une géographie, enracinée dans l'ancienne, mais différente. 
culative qui les déconcerte. On peut y voir une forme de sagesse. La revendication foncière fut pour eux plus affective, sociale ou culturelle que strictement économique.

\section{La part du tourisme}

Il existe pour Hienghène quelques projets touristiques émanant de bureaux d'études visant au développement d'activités sportives aux abords immédiats du village, comme le parapente et les sports nautiques, allant de pair avec l'aménagement de points de vue, d'aires de jeux et de camping. On songe à un accueil ultérieur de croisiéristes australiens ou de touristes japonais. Pour l'heure, quelques modestes aménagements sont visibles au centre du village, un marché, un boulodrome et un ponton d'amarrage pour l'accueil d'une quinzaine de plaisanciers. Mais le spectre des éléments valorisables à Hienghène est plus large. Le pays tout entier est un espace à découvrir. Il intègre une gamme de paysages incomparables, une nature largement intacte, une culture et une histoire particulièrement riches.

Mal dotée en terres agricoles et encore moins en surfaces pastorales, l'étroite vallée de la Hienghène compense ce handicap par une rare diversité physique associant, de Tiwandé à la Ouaièm, 25 kilomètres de littoral aux paysages contrastés, parfois sublimes, une lagune à Lindéralique avec ses roches dentelées et ses grottes, une baie sans égal ou, dans la dénomination touristique moderne, «la Poule » a prosaïquement remplacé les "Tours de Notre-Dame », une embouchure impressionnante à la Ouaiem, une belle rivière aux eaux claires dans son écrin de verdure, le charme et la fraîcheur d'un arrière-pays montagnard, quasiment vierge, allant des kaoris géants (Agathis lanceolata) du plateau de Toven jusqu'à la cime du Mont Panié (1 $630 \mathrm{~m}$ ), point culminant de l'île. Cette variété paysagère s'enrichit d'un potentiel culturel nourri de la tradition kanak, de la présence de chefferies anciennes toujours respectées, d'une histoire coloniale aux formes multiples, parfois violente, allant des santaliers et des missionnaires aux expéditions militaires, du cantonnement aux colons et aux révoltes, à travers un ensemble de lieux chargés de sens et porteurs de mémoire. Il y a, dans cette géographie totale, un gisement d'activités et d'emplois, à temps partiel ou à pleintemps, de guides-accompagnateurs, de loueurs de chevaux, de véhicules ou de bungalows, de tenanciers de gittes, d'aubergistes de brousse servant des plats locaux, sans omettre les artisans, sculpteurs, fabricants de monnaie kanak et autres agriculteurs, susceptibles de bénéficier de ces retombées touristiques. Dans cette perspective, il importe au premier chef de maintenir en bon état le patrimoine historique qui le mérite.
Ainsi du bâti trop souvent négligé. Les rares habitations coloniales encore debout et présentant un réel intérêt historique ou architectural (comme la station Laborderie à la Tipindjé dont l'état se dégrade à grande vitesse) devraient, avant qu'elles ne tombent en ruine, faire l'objet de projets de rénovation et de valorisation, en aidant financièrement leurs propriétaires actuels prêts à s'y engager. En transformant la culture locale en potentiel économique, on diversifie le tourisme, on l'amplifie, on diffuse des activités dans l'espace local et on maintient sur place les hommes et leur mémoire qui sont la substance du « pays ».

L'accueil en tribu s'inscrit dans ces perspectives. Il y a quelques années, on avait incité les Kanak à construire de petites cases traditionnelles dont existent toujours quelques spécimens dans les tribus. Elles s'accompagnaient souvent d'une restauration de type "table d'hôte ». On pouvait y prendre pension. Il s'agissait d'offrir au touriste du dépaysement et de l' " authenticité " en l'insérant au cour de la société kanak. Idée séduisante, qui s'est avérée une vue de l'esprit. À l'usage, le confort de ces cases est apparu trop sommaire pour attirer durablement des visiteurs autres que de jeunes routards avides d'expériences et au mieux de leur forme physique. Difficile, en effet, de convaincre un touriste moyen européen ou japonais, sexagénaire de préférence et pas vraiment masochiste, de l'intérêt qu'il pourrait trouver à devoir s'accroupir et ramper pour entrer dans sa hutte et à dormir sur un mince matelas de mousse ou une natte étalée à même le sol. Aussi a-t-on cessé d'édifier de telles cases au profit de bungalows plus vastes, équipés de sanitaires, mieux adaptés à la clientèle potentielle. On pourrait compléter le soutien au développement de telles initiatives en prévoyant, pour les jeunes femmes mélanésiennes, des stages d'enseignement ménager les préparant à un accueil plus " professionnel » de ces visiteurs. Le champ offert par ce tourisme vert est donc vaste et pratiquement inexploité.

\section{Les changements au village}

La fin des années 1960 marque, à Hienghène, le début de la transformation d'un village jusque là embryonnaire et léthargique. Premier signe : en 1967, à flanc de versant avec vue imprenable sur la rivière, un premier lotissement communal a été délimité. Partiellement prévu, à l'origine, pour des résidences secondaires, ce sont des habitants permanents qui viendront peu à peu s'y installer. Cinq ans plus tard, en 1973, remplaçant l'ancien bac pittoresque et désuet, un pont de béton à voie unique enjambait la Hienghène, ouvrant un accès permanent à la rive droite où se trouvaient la grande chefferie et deux hôtels et 


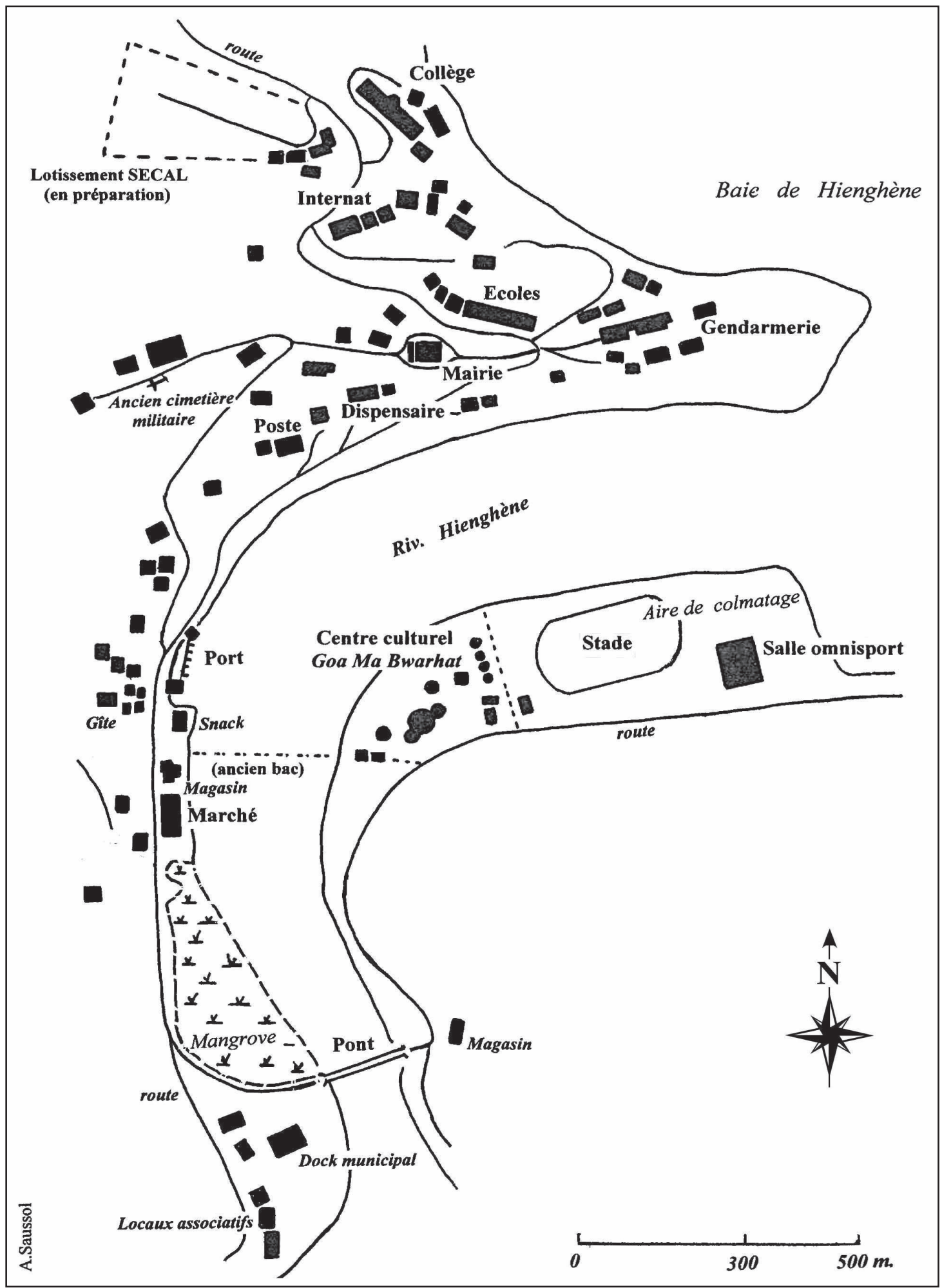

Figure 11. - Le village de Hienghène en 2004 (d'après croquis de terrain)

La croissance du village depuis 1967 est surtout le fait d'investissements de l'État (soit directs, soit transitant par les instances territoriales ou provinciales) créateurs d'emplois dans la fonction publique : Centre culturel, Services postaux, de Santé, Gendarmerie, Écoles, Collège, Internat (les Travaux publics ayant été regroupés à Touho). Peu d'initiatives privées (à part quelques commerces et l'exception notable du Relais de Koulnoué). Les fonctions du village se limitent à celles d'un petit centre administratif dispensateur de services locaux de première nécessité. Un développement à partir d'initiatives endogènes kanak s'esquisse à peine. Autre constat : aucun Kanak de Hienghène ne réside au village. Celui-ci est peuplé d'allogènes. Les natifs du pays continuent à vivre chez eux, dans les réserves (ou leurs extensions) ou sur les terres des GDPL. 
offrant un nouveau champ d'expansion au village dont il allait faciliter la fréquentation touristique. C'était le coup d'envoi de changements plus profonds.

Côté mélanésien aussi, les choses bougent. En 1977, un enfant du pays, Jean-Marie Tjibaou, est élu maire, détrônant un notable européen. Il deviendra bientôt président de l'Assemblée territoriale. Avec lui, les Kanak de Hienghène prennent le contrôle du pouvoir municipal qui va soutenir leur résurgence culturelle et politique.

En 1982-1983, sur la rive droite de la rivière, en l'honneur des deux chefferies du bord de mer et de la Chaîne, est implanté le centre culturel "Goa Ma Bwarhat " (Goa et Bouarate), support, moteur et symbole de la renaissance identitaire kanak. Une occasion pour la " coutume ", réhabilitée en tant que réalité vivante, de s’appliquer en se rénovant. Ainsi, les deux chefferies ont-elles conclu un accord sans précédent autorisant celle de la montagne, à édifier sa grande case symbolique en bord de mer face à celle de la chefferie du littoral. Le terre-plein du centre culturel entre ces deux cases, transcendé en allée cérémonielle où aucune grande manifestation n'intervient sans cérémonie d'accueil par les deux chefferies réunies, est ainsi devenu le lieu symbolique du renouveau de la coutume, de l'alliance et de l'unité de tout le pays.

Parmi les nouveaux équipements dus à la politique de rééquilibrage régional consécutive aux accords de Matignon, figurent, aux extrémités du village, deux réalisations modernes. Rive droite, près du centre culturel, sur une aire de remblaiement gagnée sur l'embouchure, un stade et une salle omnisport. Rive gauche, au-delà des anciennes écoles, le collège " Paï Kaléon " flambant neuf. Pièce maitresse du nouveau Hienghène, ce collège et son internat sont devenus une source d'activités locales. Depuis leur mise en service en 2001, ils rassemblent, de la $6^{e}$ à la $3^{\text {e, }}$, plus de 200 pensionnaires de toute la région. Enfin, entre le centre culturel et la salle omnisport, a pu être dégagée une vaste esplanade dévolue au sport, à des fêtes communales animées (avec stands de vente et de restauration pour faciliter l'écoulement des produits locaux agricoles ou artisanaux) et à l'aménagement envisagé d'un petit parc d'activités.

Au bout du compte, grâce surtout aux investissements de l'État transitant par le Territoire ou la Province, l'emploi salarié s'est développé à Hienghène. Aux emplois administratifs et municipaux s'ajoutent ceux générés par l'internat, le centre culturel et l'hôtel de Koulnoué. Des jeunes gens travaillent aussi à Nouméa ou à Koné. Le pays est vivant et les gens semblent heureux.

Une ombre vient cependant ternir cet effort d'aménagement destiné à stimuler et à soutenir un embryon de développement. Dernièrement, la commune a perdu un fleuron de son patri- moine, l'ancien poste fortifié construit en 1880 par l'infanterie de marine sur le promontoire de Kalégone. Ce fort n'était pas seulement un symbole colonial mais le témoin d'une histoire qui appartient à la mémoire kanak. Comme les rochers de la baie ou les araucarias dressés de l'ancienne chefferie, ce poste incarnait Hienghène. Un livre nourri d'extraits de correspondance de l'officier qui l'avait bâti, le capitaine Kanappe, relatait les péripéties de sa construction et de ses débuts, montrant ce qu'était Hienghène à cette époque. Il avait aussi inspiré les peintres de marine et aurait pu constituer un support essentiel pour le développement d'un tourisme culturel. Las, il n'existe plus. Dans l'indifférence générale, une institution d'État, la Gendarmerie, l'a inconsidérément rasé pour construire sur son emplacement de nouveaux locaux et d'insipides villas avec vue imprenable. Acte navrant d'autant qu'irréparable, commis sous les yeux d'un maire resté inexplicablement passif devant ce saccage de son patrimoine communal. Jean-Marie Tjibaou, homme d'intelligence et de culture qui, en d'autres temps, au nom de la mémoire kanak, avait su empêcher le dynamitage du monument de Balade, a dû se retourner dans sa tombe.

\section{BIBLIOGRAPHIE}

Courtis Christine, 1984. Après 1878 : Les souvenirs du capitaine Kanappe, Nouméa, Société d'études historiques de Nouvelle-Calédonie, Publication de la SEHNC 35, 120 p.

Duroy Lionel, 1988. Hienghène ou le désespoir calédonien, Paris, Éditions Barrault, 320 p.

Godin Patrice, 1990. Maisons, chemins, autels. La chefferie de Hienghène, in R. Boulay (éd.), De jade et de nacre, catalogue de l'exposition, Paris et Nouméa, Réunion des Musées nationaux, pp. 70-99.

-, nd. Les échanges comme poumon de la société : économies de la vie et de la puissance en pays kanak (région de Hienghène, Nouvelle-Calédonie, côte Nord-Est), mémoire de doctorat en cours, EHESs-Paris.

Saussol Alain, 1979. L'héritage. Essai sur le problème foncier mélanésien en Nouvelle-Calédonie, Paris, Société des Océanistes, Publication de la Société des Océanistes 40, 497 p. avec cartes.

—, 1989. Dynamiques foncières d'un centre de colonisation en Nouvelle-Calédonie : le pays de Hienghène, Revue française d'histoire d'Outre-Mer 284-285, pp. 455-509. 